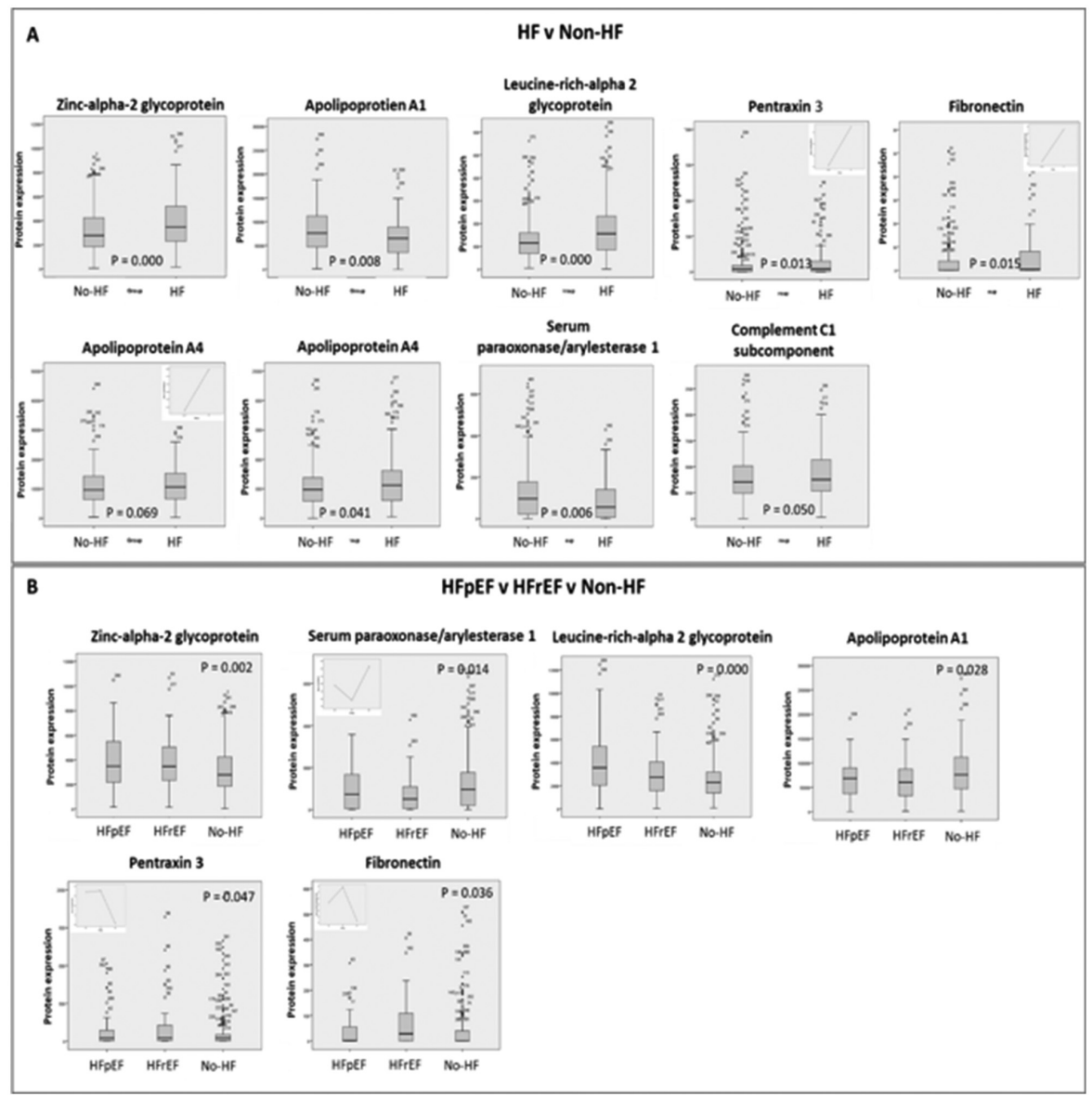

Abstract BS15 Figure 1 Box Plots of significantly changing proteins.

Figure 1 shows box plots of statistically significantly changing proteins $(p \leq 0.07)$ between HF and non-HF patients $(A)$ and $H F p E F$, HFrEF and nonHFpatients (B) at the $95 \%$ confidence level

\section{BS16 A NOVEL FLUORESCENT APELIN LIGAND TRACKS APELIN RECEPTOR INTERNALISATION}

${ }^{1}$ Elisabeth O'Flaherty*, ${ }^{1}$ Tom Williams, 'Duuamene Nyimanu, 'Robyn Macrae, ${ }^{2}$ Heather Currinn, ${ }^{2} J a s o n$ Brown, ${ }^{2}$ Alastair Brown, 'Gregory Strachan, 'Janet Maguire, ${ }^{1}$ Anthony Davenport. ${ }^{1}$ University of Cambridge; ${ }^{2}$ Sosei Heptares

\subsection{6/heartjnl-2019-BCS.179}

Introduction Fluorescent ligands are a promising tool in the study of GPCR pharmacology as they are safe and more cost effective than conventional probes such as radioligands. In this study, we investigated the use of a novel fluorescent ligand, apelin-488, to successfully show qualitative and quantitative apelin receptor internalisation, which may help further our understanding of apelin receptor pharmacology. The apelin receptor, a class A GPCR that unusually binds two endogenous peptide ligands, apelin and elabela/toddler, is expressed and functional in the cardiovascular system. Mutations in the human apelin receptor have been identified in rare diseases and our aim is to identify tool compounds to explore the potential functional consequence of these mutations.

Methods CHO-K1 cells stably expressing the wild-type apelin receptor were treated with apelin-488, fixed, and stained with Hoechst 33342 and a wheat germ agglutinin-AlexaFluor-594 conjugate as nuclear and membrane markers respectively. Triple fluorescence confocal images were generated using Opera
PhenixTM High Content Screening System and data were quantified using Harmony High Content Imaging and Analysis Software. Fluorescent ligand intensity at the membrane or in the cytoplasm was determined, providing cellular localisation for the apelin receptor following ligand binding. Activity of the fluorescent ligand at the apelin receptor in comparison to the endogenous ligand, [Pyr1]apelin-13 or CMF-019, a G protein biased small molecule apelin receptor ligand, was determined using a dynamic mass redistribution assay and radioligand binding assays.

Results The fluorescent ligand, apelin-488 binds specifically to the apelin receptor in stably transfected $\mathrm{CHO}-\mathrm{K} 1$ cells. High content screening revealed that in these cells, the apelin receptor is internalised over time and was still present at the cell membrane at an early time point, 30 secs (fig 1A). However, by 60 mins, apelin- 488 membrane localisation was reduced while internal localisation had increased, suggesting substantial apelin receptor internalisation had occurred. (fig 1B). Untransfected CHO-K1 cells showed no apelin-488 signal, supporting specific binding of the fluorescent ligand to the apelin receptor. A dynamic mass redistribution assay demonstrated that apelin-488 is functional at the apelin receptor, at a similar potency as the endogenous ligand [Pyr1]apelin-13 and the $G$ protein biased small molecule apelin receptor ligand CMF-019 (Fig 2). 